\title{
Nurse led clinics controlled hypertension and hyperlipidaemia better than usual care in diabetes
}

New JP, Mason JM, Freemantle N, et al. Specialist nurse-led intervention to treat and control hypertension and hyperlipidemia in diabetes (SPLINT): a randomized controlled trial. Diabetes Care 2003;26:2250-5.

\section{Q In patients with diabetes and high blood pressure (BP) or high cholesterol, is a nurse led hypertension or hyperlipidaemia clinic more effective than usual care for controlling BP and lipid concentrations?}

\section{METHODS}

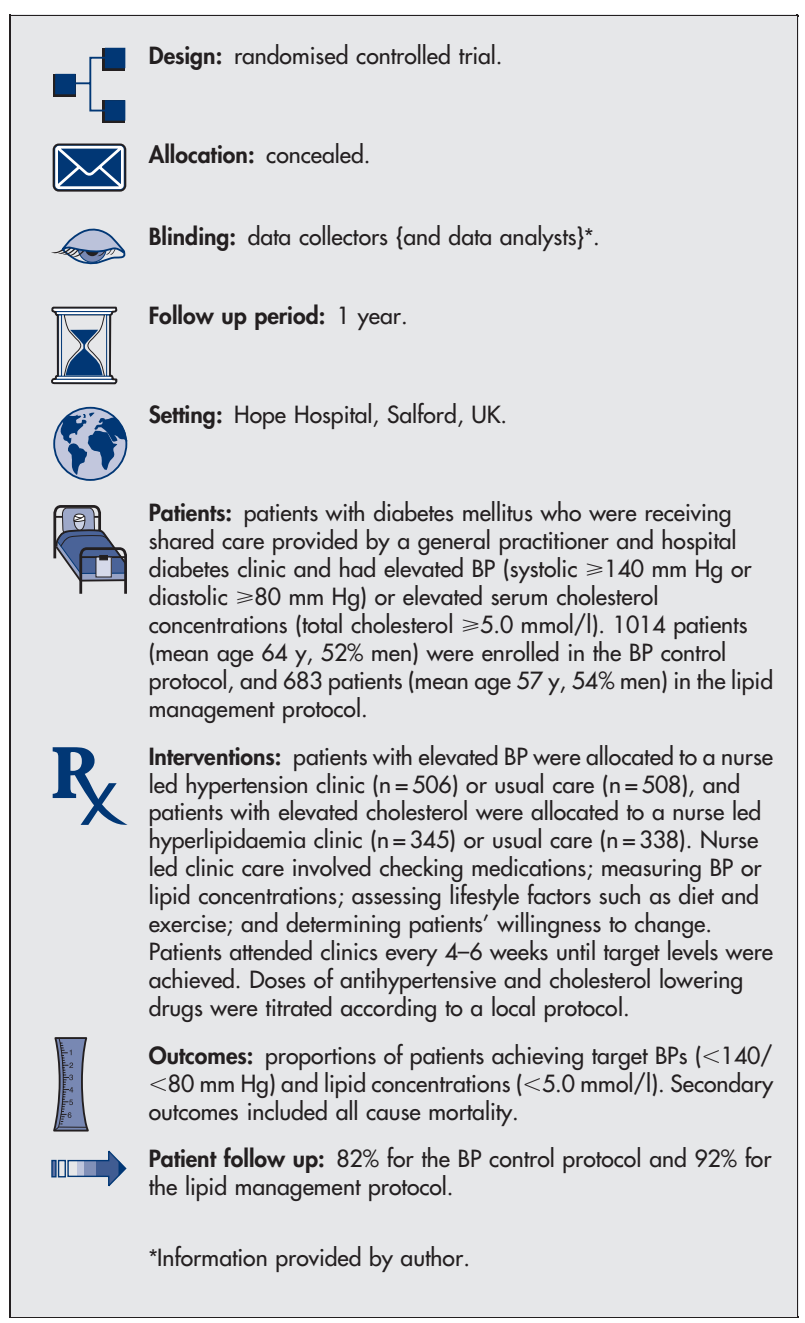

For correspondence: Dr J P New, Department of Diabetes, Hope Hospital, Salford, UK. john.new@man.ac.uk

Source of funding: Pfizer

\section{MAIN RESULTS}

Overall, more patients allocated to nurse led clinics reached target BPs and lipid concentrations (table). Analysis of only patients with hyperlipidaemia showed that more patients allocated to nurse led clinics reached target lipid concentrations (table). For patients with hypertension, the groups did not differ (table). Fewer patients who attended nurse led clinics died (table).

\section{CONCLUSION}

In patients with diabetes and high blood pressure (BP) or high cholesterol, nurse led hypertension or hyperlipidaemia clinics were more effective than usual care for achieving target BPs and lipid concentrations.

\section{Commentary}

P educing hypertension and hyperlipidaemia in patients with diabetes have been shown to reduce morbidity and mortality from cardiovascular events. ${ }^{12}$ The study by New et al considers the effectiveness of specialist nurse led interventions in achieving targets recommended by NICE and the National Service Framework for diabetes. ${ }^{34}$

Study patients were all from Salford, an inner city area with high levels of social deprivation, a fact that the authors acknowledge could have affected attendance: $16 \%$ of patients with hyperlipidaemia and $41 \%$ of patients with hypertension did not attend the nurse led clinic to which they were randomised, but returned to usual care. If they returned for annual review appointments, their results were included in the analysis.

Although more patients in the nurse led clinics achieved target BPs and lipid concentrations, results for hypertension alone were not significant. This result could have been influenced both by the high non-attendance rate in the intervention group and the 4 monthly education sessions offered to general practitioners and practice nurses providing care to patients in the usual care group.

Mortality was substantially reduced in the combined intervention group. This result could have been partly because the usual care group included more patients with a history of myocardial infarction or cerebrovascular disease, a group that is already at higher risk of mortality.

The results of this study are relevant to practice nurses, diabetic specialist nurses, and anyone providing education and pharmacological intervention to patients with diabetes. The findings add to a growing body of evidence that emphasises the importance of intensive programmes of care to assist patients in achieving recommended treatment targets, particularly when multiple risk factors are involved.

Judith A K Carrier, RGN, MSc, PGCE, Sp practitioner (PN) University of Wales College of Medicine Caerleon, South Wales, UK

BMJ 1998:317:703-13.

2 Heart Protection Study Collaborative Group. Lancet 2002;360:7-22.

3 NICE. Management of type 2 diabetes-blood pressure and blood lipids.

2002. www.nice.org.uk/cat.asp?c $=38551 /$

4 Department of Health, National Service Framework for Diabetes. Standards. 2002. www.doh.gov.uk/nst/diabetes

Nurse led blood pressure (BP) and lipid clinics $v$ usual care for patients with diabetes ${ }^{*}$

\begin{tabular}{llllll}
\hline Outcomes at 1 year & Patient group & Nurse led clinic & Usual care & RBI (95\% CI) & NNT (CI) \\
\hline BP and lipid targets achieved & All patients & $37 \%$ & $31 \%$ & $21 \%$ (6 to 39) & 16 (10 to 49) \\
BP targets achieved & Hypertension & $27 \%$ & $24 \%$ & $11 \%(-10$ to 37) & $\begin{array}{l}\text { Not significant } \\
\text { Lipid targets achieved }\end{array}$ \\
\hline & Hyperlipidaemia & $53 \%$ & $40 \%$ & $27 \%$ (7.9 to 50) & 10 (6 to 29) \\
\hline & & & & RRR (CI) & NNT (CI) \\
\hline Mortality & All patients & $3.2 \%$ & $5.7 \%$ & $44 \%$ (8 to 66) & 40 (21 to 271) \\
\hline
\end{tabular}

*Abbreviations defined in glossary; RBI, RRR, NNT, and Cl calculated from data in article. 